\title{
A Comparative Study of Face Representations in the Frequency Domain
}

\author{
Eduardo Garea Llano ${ }^{1}$, Josef Kittler ${ }^{2}$, Kieron Messer $^{2}$, and Heydi Mendez Vazquez ${ }^{1}$ \\ ${ }^{1}$ Advanced Technology Application Center. 7ma, No. 21812, Siboney, Playa, Cuba, 12200 \\ \{egarea, hmendez\} @cenatav.co.cu \\ ${ }^{2}$ Centre for Vision Speech and Signal Processing, University of Surrey, GU 7HX, UK \\ \{j.kittler, k.messer\}@eim.surrey.ac.uk
}

\begin{abstract}
The variation of illumination of an object can produce large changes in the image plane, significantly impairing the performance of face verification algorithms. In this paper we present a comparison of several face representation methods from the point of view of their sensibility to illumination changes. The sensibility is measured in term of the overlap of distribution of normalized correlations for inter class and intra class image comparison. We compared a combination of differentiated image in the frequency domain and the performance of Fourier parameters to obtain an illumination insensitive representation. The result suggests, that better illumination invariance could be achieve in feature spaces developed for a differentiated image rather than using the original input image.
\end{abstract}

\section{Introduction}

A number of algorithms [1-4] have been proposed for the face recognition problem, to compensate for illumination changes. In general the variations between images of different faces are smaller than that of the same face taken in a variety of environments. External factors such as pose and illumination can cause different conditions and significant changes in the image plane. It has been shown that illumination causes larger variation in face images than pose [2]. The importance of illumination is further illustrated by examination of the eigenface method [5]. Belhumeur improved the accuracy of a recognition system based on eigenfaces, by removing the first three principal components [4].

In recent years many appearance-based algorithms have been proposed to deal with the problem [7-10]. Belhumeur showed [7], that the set of images of an object in fixed pose but under varying illumination forms a convex cone in the space of images. This method requires a large amount of training data, but Lee showed that the subspace could be generated using only nine images captured under a particular set of illumination conditions [10] Recognition is carried out by finding the distance of the probe image to the illumination cone. These algorithms work well, but are computationally expensive.

Wei and Lai [11] showed that the facial edge feature is an important cue for face recognition and is less sensitive to illumination changes. Barnabas [12] used the Hausdorff distance between the facial edge maps for face recognition. Gao and Leung 
[13] furthermore encode the edge map (LEM) by a polygonal line fitting process and computed the similarity of two LEMs with a line segment Hausdorff distance. Savvides [14] showed that modeling the complex phase spectrum in the frequency domain produces a face representation that is tolerant to illumination variations, and also can automatically handle occlusions (i.e. missing features) without any special preprocessing.

In this paper we exploit the fact that although the actual intensity value at each image point is affected by illumination changes, the local differences are much more stable. We propose a novel representation in the frequency domain which is derived from a complex input image with the real and imaginary parts defined by the $\mathrm{x}$ and $\mathrm{y}$ derivatives of the original image. The sensitivity to illumination is compared with that of the original image and features from the Fourier spectrum.

The contrast XM2VTS database [15] and the Yale B face database [16] are used in the experiments. The images in the XM2VTS database were captured in a controlled environment in which illumination variations are minimized in one set of images but accentuated across another set.

The Yale B database contains images under widely varying illumination conditions and poses of ten subjects. Tests were carried out using the frontal pose set of images with varying illumination

We show that the Real Part of the Fourier transform from differentiated complex image yield the most consistent results across all experimental combinations and can be used as an illumination insensitive representation for face verification.

In the next section, we describe the four representations used in the comparative study. Section 3 details the experimental procedures to obtain and compare the representations. The results of the experiments are presented in Section 4 and we conclude in Section 5.

\section{Representations}

In this section we describe four face representations that will be compared to identify the most illumination insensitive. They are defined in the frequency domain (magnitude, phase angle, real part and imaginary part) computed from differentiated face images forming a complex input image.

\subsection{Fourier Transform}

The Fourier transform produces a representation of a signal, in term of a weighted sum of complex exponentials. The defining formulas for the forward Fourier and the inverse Fourier transforms are as follows. Given an image $a$ its forward discrete Fourier transform $A$ is defined as

$$
A(\Omega, \Psi)=\sum_{m=-\infty}^{+\infty} \sum_{n=-\infty}^{+\infty} a[m, n] e^{-j(\Omega m+\Psi n)}
$$

and vice versa, its inverse as 


$$
a[m, n]=\frac{1}{4 \pi^{2}} \int_{-\pi-\pi}^{+\pi+\pi} \int_{-\pi} A(\Omega, \Psi) e^{+j(\Omega m+\Psi n)} d \Omega d \Psi
$$

The Fourier spectrum is the complex function of the real frequency variables and it can be represented by in its real and imaginary parts. Alternatively we can represent it by its magnitude and phase as:

$$
A(\Omega, \Psi)=|A(\Omega, \Psi)| e^{j \varphi(\Omega, \Psi)}
$$

Where $|A(\Omega, \Psi)|$ is the magnitude and $(\Omega, \Psi)$ is the phase corresponding to frequency $\operatorname{pair} \varphi(\Omega, \Psi)$.

\subsection{Derivatives of Image}

Illumination is a low frequency phenomenon the effect of which can be suppressed by differentiation.

In computer vision, image differentiation is traditionally implemented by convolving the signal with some form of linear filter, usually a filter that approximates a first or second derivative operator. An odd symmetric filter will approximate a first derivative.

The gradient of the image is a vector with the derivatives in $\mathrm{x}$ and $\mathrm{y}$ directions as its components, i.e.

$$
\begin{aligned}
& \Delta_{x}(x, y)=\frac{a\left(x+d_{x}, y\right)-a(x, y)}{d x} \\
& \Delta y(x, y)=\frac{a\left(x+d_{y}+y\right)-a(x, y)}{d y}
\end{aligned}
$$

Rather than in a vector form it is convenient to represent the derivatives in $\mathrm{x}$ and $\mathrm{y}$ directions as a complex image

$$
g(x, y)=\Delta_{x}(x, y)+j \Delta_{y}(x, y)
$$

\section{Experimental Methods}

The basic idea of the proposed experimental method is shown in Fig. 1. The principal representation scheme consists in the combination of the elements that we described above. We use the complex first derivative image to highlight frequency content and, then transform it to the frequency domain and determine its characteristics. These characteristics computed for a pair of images will be compared using normalized correlation defined as: 


$$
N c=\frac{\sum_{i} \sum_{j}\left(w(i, j) w_{1}(i, j)\right)}{\sqrt{\sum_{i} \sum_{j} w(i, j)^{2} \sum_{i} \sum_{j} w_{1}(i, j)^{2}}}
$$

Where the first image is $w(i, j)$, the second image is $w_{1}(i, j)$. The idea is to measure how their similarity is affected by illumination changes. Normalized correlation has been chosen as it has proved to be a successful similarity measure in face recognition. For identical images it takes the maximum value equal to unity. Examples of generated representations by principal scheme are shown in Fig 2.

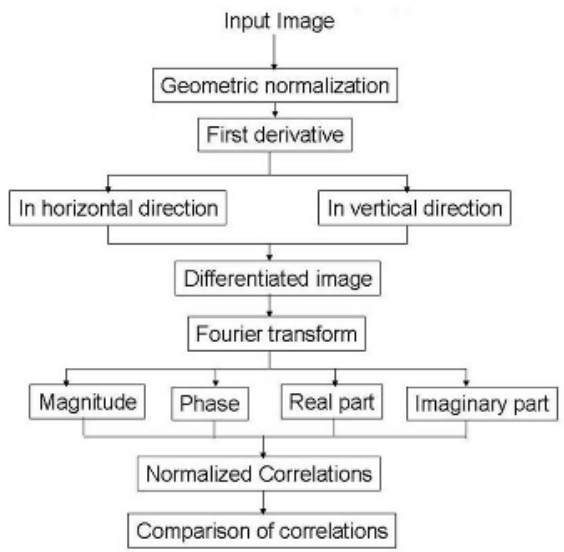

Fig. 1. The flow diagram of the principal proposed experimental method

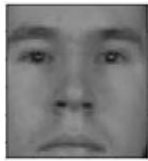

a

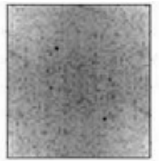

b

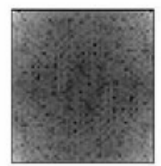

f

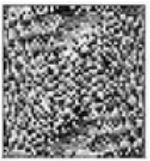

c

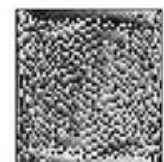

g

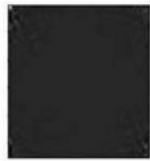

d

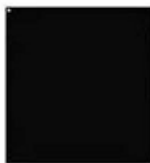

h

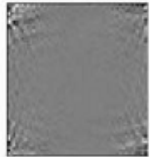

e

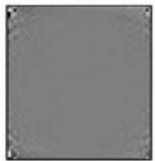

i

Fig. 2. Image Representations used in the comparative study: a)Geometric normalized input image, b)Magnitude, c)Phase, d)Real part, e) Imaginary part of the frequency spectrum of the differentiated image. f)Magnitude, g)Phase, h)Real part, i) Imaginary part of the frequency spectrum of the input image.

In the second representation scheme the objective is to transform the input image to the frequency domain directly and determine its characteristics. 
The third experimental scheme is very simple. It directly involves the original input images geometrically normalized as in the first and second experimental schemes.

\subsection{Geometric Normalization Algorithm}

The geometric normalization used consists of the following steps: Smoothing, rotating, scaling and resampling the input image. The smoothing is performed by convolution with a Gaussian Filter of size $5 \times 5$.

The rotation operation is designed to ensure that the line connecting the centres of the two eyes is parallel to the $\mathrm{x}$ - axis. The image scaling and resampling scaling outputs an image of size 55 rows x 51 columns. The left-eye is mapped onto the pixel position $(19,38)$ and the right-eye is mapped onto the pixel position $(19,12)$, Fig. 3.

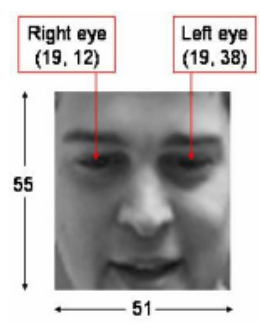

Fig. 3. Geometric normalized image

For a thorough evaluation of the proposed representations, we calculated and compared the normalized correlations using images from the XM2VTS database and Yale B database.

\subsection{The XM2VTS Database}

For a thorough evaluation of the proposed method, we calculated and compared the normalized correlations using images from the XM2VTS database (Fig. 4).

The XM2VTS database contains images of 295 subjects captured over 4 sessions in a controlled uniformly illuminated environment and with illumination from left and right over 2 sessions. The database uses a standard protocol [15].

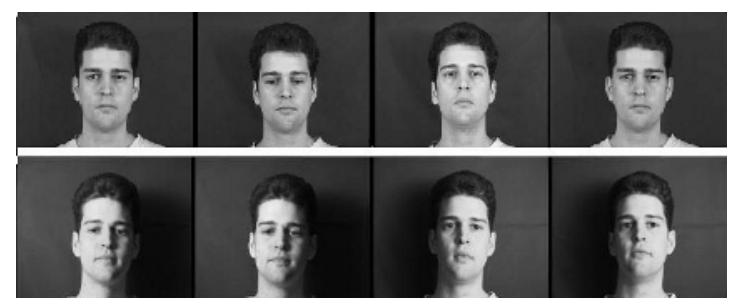

Fig. 4. Examples of the XM2VTS database: Normal illuminated (top), Illuminated from left and right (bottom) 
We also used a derivative database obtained by photometric normalization of images of the XM2VTS. The images were normalized using the anisotropic smoothing method [17].

\subsection{The Yale B Database}

The Yale B database contains 64 different illumination conditions for 10 subjects. The illumination conditions are a single light source, the position of which varies horizontally (from $-130^{\circ}$ to $130^{\circ}$ ) and vertically (from $-40^{\circ}$ to $65^{\circ}$ ). We take 4 images per each subject with different illumination condition and we also used a derivative database obtained by photometric normalization of one image per person in the Yale B database. The images were normalized using the anisotropic smoothing method [17] (Fig.5).

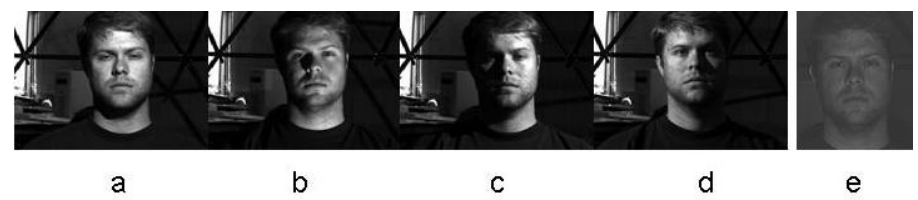

Fig. 5. Examples of the Yale B database: a,b,c,d., illuminated images from above, below, right and left respectively; e, photometrically normalized image

\subsection{Comparison of Normalized Correlations}

In the XM2VTS database the normalized correlations were compared in two different combinations for each database

- A: 295 subjects of the XM2VTS database against the same subjects using different images for each subject

- B: 295 subjects of the XM2VTS database against 294 different subjects using different images for each subject.

Correlations were carried out among normal illuminated, illuminated from right and left, and photometric normalized images from XM2VTS database to verify in many different conditions the results of comparisons.

In the YaleB database the normalized correlations were compared in two different combinations.

- A: 64 subjects of the Yale B database against the same subjects using different images for each subject

- B: 64 subjects of the Yale B database against 63 different subjects using different images for each subject.

Correlations were carried out among, illuminated from right, left, above and bellow, and photometric normalized images to verify in different conditions the results of comparisons.

In table 1 we show the different variants of normalized correlations and its comparison. 


\section{Experimental Results}

This section presents a summary of the results of the comparison of the proposed representations by the experimental schemes described.

First, the results of comparisons 1, 2 and 3 from Table 1 are presented in Table 2. We can see that the real part and imaginary part obtained by principal representation scheme are the best spectral properties that reflect the similarity between different images of the same subject and the dissimilarity between images of different subjects taken under controlled illumination conditions of XM2VTS database. The original image in this case best reflects the similarity under controlled normal illumination conditions.

Results on the Yale B database shows that the real part obtained by the principal experimental scheme is consistent representation with high correlation coefficients between images of the same subject taken in different illumination conditions and photometrically normalized.

The second experiment relates to the comparisons 4 and 5 from Table 1 . The results are shown in Table 3. The experiment shows that the real part obtained by the principal experimental scheme is consistent representation with high correlation coefficients between images of the same subject taken in normal condition and images taken in different illumination conditions (Fig. 6) and photometrically normalized in both databases.

Table 1. Comparison of normalized correlations

\begin{tabular}{|c|c|l|l|l|}
\hline No & $\begin{array}{l}\text { Comparison } \\
\text { of correla- } \\
\text { tions }\end{array}$ & $\begin{array}{l}\text { Compared images } \\
\text { (XM2VTS database) }\end{array}$ & \multicolumn{1}{|c|}{$\begin{array}{l}\text { Compared images } \\
\text { (Yale B database) }\end{array}$} & $\begin{array}{l}\text { Compared } \\
\text { represen- } \\
\text { tations }\end{array}$ \\
\hline 1 & A vs. B & $\begin{array}{l}\text { Normally illuminated } \\
\text { vs. normal illuminated } \\
\text { (8 by 8 images) }\end{array}$ & $\begin{array}{l}\text { Illuminated from right } \\
\text { and left vs. Illumi- } \\
\text { nated from right and } \\
\text { left (2 by 2 images) }\end{array}$ & $\begin{array}{l}\text { Principal, } \\
\text { Second, } \\
\text { Third }\end{array}$ \\
\hline 2 & A vs. B & $\begin{array}{l}\text { Illuminated from right } \\
\text { and left vs. Illumi- } \\
\text { nated from right and } \\
\text { left (4 by 4 images) }\end{array}$ & $\begin{array}{l}\text { Illuminated above and } \\
\text { bellow vs. illuminated } \\
\text { above and bellow (2 } \\
\text { by 2 images) }\end{array}$ & $\begin{array}{l}\text { Principal, } \\
\text { Second, } \\
\text { Third }\end{array}$ \\
\hline 3 & A vs. B & $\begin{array}{l}\text { Normally illuminated } \\
\text { vs. illuminated from } \\
\text { right and left (8 by 4 } \\
\text { images) }\end{array}$ & $\begin{array}{l}\text { Illuminated from right } \\
\text { and left vs. illumi- } \\
\text { nated above and bel- } \\
\text { low (2 by 2 images) }\end{array}$ & $\begin{array}{l}\text { Principal, } \\
\text { Second, } \\
\text { Third }\end{array}$ \\
\hline 4 & A vs. A & $\begin{array}{l}\text { Normally illuminated } \\
\text { vs. photometrically } \\
\text { normalized (2 by 8) } \\
\text { images }\end{array}$ & $\begin{array}{l}\text { Illuminated from right } \\
\text { and left vs. photomet- } \\
\text { rically normalized (1 } \\
\text { by 2 images) }\end{array}$ & Principa1 \\
\hline 5 & A vs. A & $\begin{array}{l}\text { Illuminated from right } \\
\text { and left vs. photomet- } \\
\text { rically normalized (4 } \\
\text { by 2 images) }\end{array}$ & $\begin{array}{l}\text { Illuminated above and } \\
\text { bellow vs. photomet- } \\
\text { rically normalized (1 } \\
\text { by 2 images) }\end{array}$ & Principa1 \\
\hline
\end{tabular}


Table 2. Principal results of comparison between normalized correlations (1,2 and 3; Table 1)

\begin{tabular}{|c|c|c|c|c|c|c|c|c|c|}
\hline \multirow[t]{2}{*}{$\mathrm{Rp}$} & \multirow[t]{2}{*}{ Par } & \multicolumn{4}{|c|}{ XM2VTS database } & \multicolumn{4}{|c|}{ Yale B database } \\
\hline & & $\begin{array}{l}\mathrm{Av} \\
\text { (A) }\end{array}$ & $\begin{array}{l}\mathrm{Av} \\
\text { (B) }\end{array}$ & $\begin{array}{l}\text { overlap } \\
\%\end{array}$ & Dist & $\begin{array}{l}\mathrm{Av} \\
\text { (A) }\end{array}$ & $\begin{array}{l}\mathrm{Av} \\
(\mathrm{B})\end{array}$ & $\begin{array}{l}\text { overlap } \\
\%\end{array}$ & Dist \\
\hline \multicolumn{2}{|c|}{ Comparison } & \multicolumn{4}{|c|}{1} & \multicolumn{4}{|c|}{1} \\
\hline \multirow[t]{4}{*}{ Pr. } & $\mathrm{Mg}$ & 0.69 & 0.63 & 81.70 & 0.06 & 0.78 & 0.73 & 93.58 & 0.05 \\
\hline & $\mathrm{Fi}$ & 0.12 & 0.02 & 12.88 & 0.09 & 0.09 & 0.04 & 44.44 & 0.05 \\
\hline & $\mathbf{R}$ & 0.83 & 0.67 & 10.17 & 0.16 & 0.92 & 0.56 & 22.88 & 0.36 \\
\hline & I & 0.57 & 0.25 & 13.83 & 0.32 & 0.52 & 0.51 & 95.00 & 0.01 \\
\hline \multirow[t]{4}{*}{ Sec. } & $\mathrm{Mg}$ & 0.73 & 0.69 & 63.73 & 0.03 & 0.75 & 0.70 & 93.33 & 0.05 \\
\hline & $\mathrm{Fi}$ & 0.14 & 0.04 & 64.41 & 0.10 & 0.13 & 0.02 & 58.55 & 0.11 \\
\hline & $\mathrm{R}$ & 0.99 & 0.98 & 22.03 & 0.01 & 0.97 & 0.95 & 44.06 & 0.02 \\
\hline & I & 0.63 & 0.35 & 20.00 & 0.27 & 0.58 & 0.33 & 18.51 & 0.25 \\
\hline Th. & Or & 0.76 & 0.40 & 4.40 & 0.36 & 0.45 & 0.34 & 75.55 & 0.11 \\
\hline \multicolumn{2}{|c|}{ Comparison } & \multicolumn{4}{|c|}{2} & \multicolumn{4}{|c|}{2} \\
\hline \multirow{4}{*}{ Pr. } & $\mathrm{Mg}$ & 0.72 & 0.66 & 50.84 & 0.09 & 0.72 & 0.66 & 50.84 & 0.09 \\
\hline & $\mathrm{Fi}$ & 0.13 & 0.03 & 98.64 & 0.1 & 0.13 & 0.03 & 98.64 & 0.1 \\
\hline & $\mathbf{R}$ & 0.90 & 0.77 & 20.00 & 0.23 & 0.93 & 0.69 & 20.86 & 0.24 \\
\hline & I & 0.75 & 0.32 & 19.15 & 0.43 & 0.69 & 0.63 & 91.30 & 0.06 \\
\hline \multirow[t]{4}{*}{ Sec. } & $\mathrm{Mg}$ & 0.80 & 0.75 & 58.64 & 0.07 & 0.82 & 0.73 & 75.39 & 0.09 \\
\hline & $\mathrm{Fi}$ & 0.21 & 0.07 & 85.42 & 0.14 & 0.17 & 0.08 & 54.41 & 0.09 \\
\hline & $\mathrm{R}$ & 0.99 & 0.98 & 61.69 & 0.01 & 0.95 & 0.93 & 71.56 & 0.02 \\
\hline & $\mathrm{I}$ & 0.96 & 0.89 & 67.00 & 0.07 & 0.93 & 0.78 & 69.00 & 0.15 \\
\hline Th. & Or & 0.89 & 0.82 & 64.00 & 0.07 & 0.85 & 0.77 & 73.14 & 0.08 \\
\hline \multicolumn{2}{|c|}{ Comparison } & \multicolumn{4}{|c|}{3} & \multicolumn{4}{|c|}{3} \\
\hline \multirow[t]{4}{*}{ Pr. } & $\mathrm{Mg}$ & 0.68 & 0.65 & 86.39 & 0.04 & 0.63 & 0.58 & 85.50 & 0.05 \\
\hline & $\mathrm{Fi}$ & 0.12 & 0.05 & 57.82 & 0.05 & 0.10 & 0.03 & 56.79 & 0.07 \\
\hline & $\mathbf{R}$ & 0.82 & 0.63 & 32.24 & 0.19 & 0.90 & 0.65 & 31.35 & 0.25 \\
\hline & I & 0.57 & 0.48 & 58.50 & 0.09 & 0.53 & 0.45 & 57.45 & 0.08 \\
\hline \multirow[t]{4}{*}{ Sec. } & $\mathrm{Mg}$ & 0.78 & 0.67 & 68.00 & 0.11 & 0.81 & 0.69 & 67.58 & 0.12 \\
\hline & $\mathrm{Fi}$ & 0.25 & 0.03 & 12.00 & 0.22 & 0.15 & 0.08 & 40.00 & 0.08 \\
\hline & $\mathrm{R}$ & 0.99 & 0.99 & 100.00 & 0.00 & 0.97 & 0.93 & 75.00 & 0.04 \\
\hline & $\mathrm{I}$ & 0.70 & 0.55 & 56.32 & 0.15 & 0.68 & 0.62 & 65.52 & 0.06 \\
\hline Th. & Or & 0.60 & 0.62 & 55.00 & 0.02 & 0.57 & 0.53 & 53.65 & 0.04 \\
\hline
\end{tabular}

Table 3. Result of comparisons between normalized correlations (4 and 5, Table 1)

\begin{tabular}{|c|c|c|c|c|}
\hline \multirow{2}{*}{ Par } & \multicolumn{2}{|c|}{ XM2VTS database } & \multicolumn{2}{c|}{ Yale B database } \\
\cline { 2 - 5 } & Av(A) & $\operatorname{Av}(\mathrm{A})$ & $\mathrm{Av}(\mathrm{A})$ & $\mathrm{Av}(\mathrm{A})$ \\
\hline Comparison & \multicolumn{2}{|c|}{$\mathbf{4}$} & \multicolumn{2}{c|}{$\mathbf{4}$} \\
\hline $\mathrm{Mg}$ & 0.63 & 0.67 & 0.56 & 0.58 \\
\hline $\mathrm{Fi}$ & 0.09 & 0.18 & 0.11 & 0.16 \\
\hline $\mathbf{R}$ & $\mathbf{0 . 7 1}$ & $\mathbf{0 . 7 9}$ & $\mathbf{0 . 8 5}$ & $\mathbf{0 . 8 9}$ \\
\hline $\mathrm{I}$ & 0.46 & 0.61 & 0.51 & 0.63 \\
\hline Comparison & \multicolumn{3}{|c}{$\mathbf{5}$} & \multicolumn{2}{|c|}{$\mathbf{~}$} \\
\hline $\mathrm{Mg}$ & 0.56 & 0.57 & 0.63 & 0.67 \\
\hline $\mathrm{Fi}$ & 0.12 & 0.13 & 0.08 & 0.11 \\
\hline $\mathbf{R}$ & $\mathbf{0 . 8 1}$ & $\mathbf{0 . 8 3}$ & $\mathbf{0 . 9 2}$ & $\mathbf{0 . 9 6}$ \\
\hline $\mathrm{I}$ & 0.43 & 0.64 & 0.45 & 0.67 \\
\hline
\end{tabular}



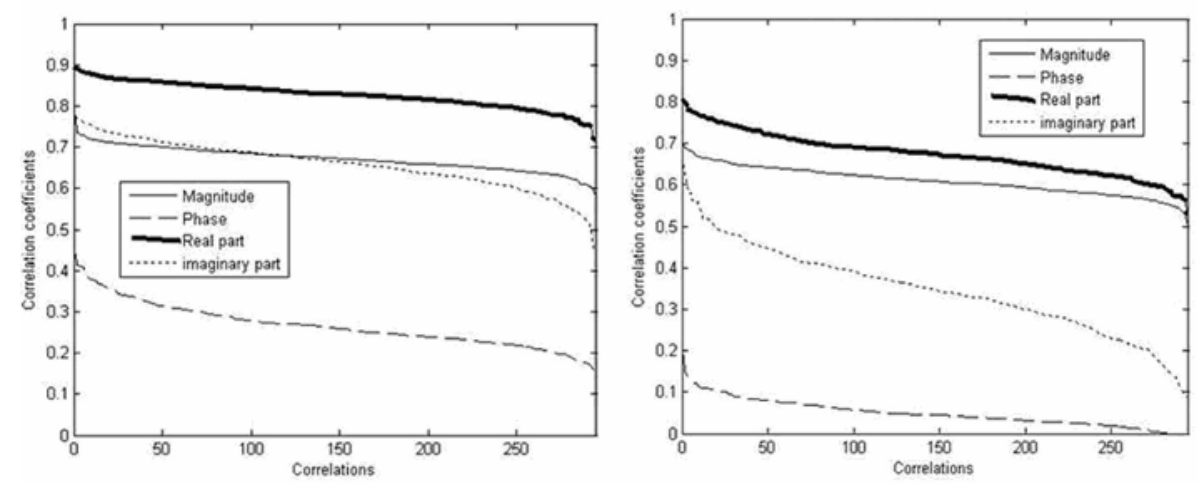

Fig. 6. Results of normalized correlation A (left) and B(right) using normally illuminated and illuminated images from right and left in the XM2VTS database by principal experimental scheme

\section{Conclusions}

We proposed a novel face image representation in the frequency domain which is derived from a complex input image with the real and imaginary parts defined by the $\mathrm{x}$ and $y$ derivatives of the original image. The experimental results show that the real part obtained by the proposed experimental scheme is the most consistent representation with high correlation coefficients between images of the same subject taken in normal condition and images taken in different illumination conditions.

In tables 2 and 3: Mg- Magnitude, Fi- Phase, R-Real part, I- Imaginary part, Or: original image, Pr, Sec. and Th.: Principal, Second and Third representation schemes respectively, $\operatorname{Av}(\mathrm{A}), \operatorname{Av}(\mathrm{B})$ : mean of correlation coefficients, overlap\%: Percent of overlapping of compared correlations, Distance: Difference between $\operatorname{Av}(A)$ and $\operatorname{Av}(B)$.

\section{References}

1. Acton, S.: Multigrid Anisotropic Diffusion. IEEE Trans. Image Processing, vol. 7, 1998

2. Adini, Y., Moses, Y. and Ullman, S.: Face recognition: the problem of compensating for illumination changes. IEEE Trans. Pattern Anal. Mach. Intelligence, pp721- 732, (1997)

3. Bailly-Bailliere, E.: The BANCA Database and Evaluation Protocol AVBPA, (2003)

4. Belhumeur, P.,. Hespanha, J., Kriegman, D.: Eigenfaces vs. Fisherfaces: Recognition using Class Speci.c Linear Projection. IEEE Trans. Pattern Anal. Mach. Intelligence, vol. 19, pp711-720, (1997).

5. Turk, M., Pentland, A.: Eigenfaces for Recognition. J. Congitive Neuroscience, vol. 3, pp 71-86, (1991)

6. Belhumeur, P., Hespanha, J.P: Eigenfaces vs. Fisherfaces: Recognition using class speci_c linear projection. IEEE PAMI 19, 711-720, (1997)

7. Belhumeur, P., D. Kriegman,: What is the set of images of an object under all possible lighting conditions. Int. J. of Computer Vision 28, 245-260(1998) 
8. Georghiades, A., Kriegman, D., Belhumeur, P.: From few to many: Generative models for recognition under variable pose and illumination. IEEE PAMI (2001)

9. Riklin-Raviv, T.,., Shashua, A.: The Quotient image: class-based re-rendering and recognition with varying illumination conditions. In: IEEE PAMI. (2001)

10. Lee, K., Ho, J., Kriegman, D.: 9 Points of Light: Aquiring Subspaces for Face Recognition Under Variable Lighting IEEE Proc. Conf. Computer Vision and Pattern Recognition, (2001).

11. Shou Der Wei and Shang-Hong Lai: Robust Face Recognition under lightinng Variations, Proc. Conf on Pattern Recognition IEE (2004).

12. Barnamas, T.: Comparing face images using the modified Hausdorff distance. Pattern Recognition. Vol. 31, No.12 pp. 1873-1881, (1991).

13. Gao, Y., Leung, K.: Face recognition using line edge map. IEEE Trans. Pattern Analysis Mach. Intel., Vol. 24, No. 6 pp. 764-779, (2002).

14. Savvides, M., Vijaya Kumar, BVK.and Khosla, P.K.: Eigenphases vs. Eigenfaces Proc. Conf on Pattern Recognition, IEEE (2004).

15. Luettin, J. and Maitre, G.: Evaluation Protocol for the XM2FDB Database, (IDIAP, 1998).

16. The Yale Face Database, URL: http://cvc.yale.edu/projects/yalefaces/yalefaces.html.

17. Short, J., Kittler, J. and Messer, K.: A comparison of photometric normalization algorithms for face verification, AVBPA 2005: 617-626, (2004) 